Research Paper

\title{
Effect of Sucrier Banana Peel Extracts on Inhibition of Melanogenesis through the ERK Signaling Pathway
}

\author{
Naphichaya Phacharapiyangkul ${ }^{1}$, Krit Thirapanmethee ${ }^{1}$, Khanit Sa-ngiamsuntorn², Uraiwan Panich ${ }^{3}$,
} Che-Hsin Lee ${ }^{4} 5^{\boxplus}$, Mullika Traidej Chomnawang ${ }^{1 凶}$

1. Department of Microbiology, Faculty of Pharmacy, Mahidol University, Bangkok, Thailand

2. Department of Biochemistry, Faculty of Pharmacy, Mahidol University, Bangkok, Thailand

3. Department of Pharmacology, Faculty of Medicine Siriraj Hospital, Mahidol University, Bangkok, Thailand

4. Department of Biological Sciences, National Sun Yat-sen University, Kaohsiung, Taiwan

5. Department of Medical Research, China Medical University Hospital, China Medical University, Taichung 404, Taiwan

$\triangle$ Corresponding authors: Mullika T Chomnawang, Department of Microbiology, Faculty of Pharmacy, Mahidol University, 447 Sri Ayudthaya Road, Rachathevi, Bangkok 10400, Thailand, Tel +66 2644868090 ext 5509, Fax +66 2644 8692, Email: mullika.tra@mahidol.ac.th or Dr. Che-Hsin Lee, Department of Biological Sciences, National Sun Yat-sen University, Kaohsiung, Taiwan, 70 Lienhai Rd. Kaohsiung 80424, Taiwan. E-mail: chlee@mail.nsysu.edu.tw

(c) Ivyspring International Publisher. This is an open access article distributed under the terms of the Creative Commons Attribution (CC BY-NC) license (https:// creativecommons.org/licenses/by-nc/4.0/). See http://ivyspring.com/terms for full terms and conditions.

Received: 2018.12.09; Accepted: 2019.03.23; Published: 2019.04.25

\begin{abstract}
Hyperpigmentation is a type of pigmentary disorder induced by overexpression of melanin content activated severe esthetic problems as melasma, freckle, ephelides, lentigo and other forms on human skin. Several whitening agents have restricted use because of their side effects or stability such as kojic acid, ascorbic acid and hydroquinone can act as cytotoxic substance which associated to dermatitis and skin cancer. To find for the safe substance, this study aimed to find for the ability of several components in Sucrier banana peel (SBP) extracts to inhibit melanogenesis process through p38 signaling pathway in B16F10 mouse melanoma cells. Tyrosinase activity and the cellular melanin content were dose dependent manner decreasing after SBP treatment. Furthermore, SBP decreased the expression of melanogenesis relate protein as microphthalmia-associated transcription factor (MITF) and tyrosinase protein after 24 hours incubation with $\alpha$-melanocyte stimulating hormones (MSH) stimulating. The findings demonstrated that SBP contained an effective agent for hyperpigmentation inhibitor through p38 signaling pathways without any effect to ERK pathway, and subsequent down-regulate MITF expression and tyrosinase enzyme family production.
\end{abstract}

Key words: sucrier banana peel; phenolic compounds; tyrosinase; microphthalmia-associated transcription factor; melanogenesis

\section{Introduction}

Melanin is produced within melanosome which is an important organelle inside the melanocyte cells. Melanocyte is located in the basal layer of skin epidermis. After production process, melanin is pigment responsible for skin, hair and eye coloration which function as naturalize antioxidant, detoxification agent, and powerful-cation chelator also can act as universal protection against UV damage [1]. Abnormal melanin production can cause pigmentary disorder such as hypopigmentation and hyperpigmentation. Hypopigmentation causes vitiligo, albinism and abnormal hair problems while hyperpigmentation causes freckles, melasma, age spot and post-inflammatory hyperpigmentation. Genetic predisposition, hormonal changes especially estrogen also others such as liver disease, tumor, cancer, malnutrition or irregular function of pituitary gland are the intrinsic factors including extrinsic factor likes UV exposure, toxic substances are the major cause of hyperpigmentation [2].

Tyrosinase is a rate limiting step enzyme that targets the process of both stimulating and inhibiting the melanin production process. Melanin stimulation is used as a tanning agent or hair depigmentation treatment. Tyrosinase expression is controlled by microphthalmia-associated transcription factor 
(MITF) which is melanocyte-specific transcription factor that controlled melanocyte differentiation, proliferation and survival $[3,4]$. The responsibility of MITF to melanogenisis is to increase tyrosinase expression by binding to DNA in the structure of homodimer or hetero dimer with MiT protein, these binding site involving E-Box and M-Box flanking thymidine nucleotide which stimulates the continuously steps to melanogenic enzyme production such as tyrosinase-related protein 1 (TRP-1), tyrosinaserelated protein-2 (TRP-2) and dopachrome tautomerase. Regulation of MITF starts from signal transduction after a-MSH bind to MC1R receptor which stimulates mitogen-activated protein kinase (MARKs) which is serine/threonine kinase and also include extracellular signaling-regulated kinase (ERK) and also p38 as well. Several studies found that melanin synthesis is controlled by several signaling pathways such as phosphotidylineasital-3-kinase (PI3K/AKT) can be suppressed by natural substances which contain polyphenol group through ERK signaling pathway of B16F10 mouse melanoma cells $[5,6]$.

This study aimed to find for the effect on melanogenesis inhibition from Sucrier banana peel (SBP) extracts. SBP may contain several types of polyphenolic compounds that act as tyrosinase inhibitor such as catechin, procyanidin, ferullic acid, gallic acid which detected by expression of ERK signaling pathway that effects the production of melanogenic enzymes such as TRP-1 and TRP-2 [7-10].

\section{Materials and methods}

\section{Chemicals and Materials}

Totally ripped (7 days after harvesting) Sucrier banana (musa spp.) peels from Kampangpetch Province, Thailand. Washed banana peels with water then sun-baked until totally dried, grinded by blender and extracted by soaking with $60 \%$ methanol for 24 hours (MW24), centrifuged with 95\% ethanol for 10 minutes at $4{ }^{\circ} \mathrm{C}(\mathrm{E} 4)$, boiling in DI water at $60{ }^{\circ} \mathrm{C}$ for 20 minutes (W60) and boiling in $95 \%$ methanol at $60{ }^{\circ} \mathrm{C}$ for 20 minutes (M60). Next step was to decrease the volume with rotary evaporator at $40{ }^{\circ} \mathrm{C}$ and was to dry by freeze dryer Christ Alpha- 4 LD plus.

\section{Cell culture}

B16F10 mouse melanoma cells were cultivated in Dulbecco's modified Eagle's medium (DMEM) supplemented with $10 \%$ fetal bovine serum at $37^{\circ} \mathrm{C}$ in an incubated with $5 \% \mathrm{CO}_{2}$. After 24 hours incubation, then changed the medium to serum free media with difference concentration of Sucrier banana peel (SBP) extracts with a-MSH stimulation. Then cells were harvested by trypsinization for the next experiments.

\section{Cell viability assay}

Cytotoxicity of SBP extracts was measured by MTT assay. The cells $\left(5 \times 10^{3}\right)$ were seeded to 96 well plate and cultivated in DMEM for 24 hours at $37^{\circ} \mathrm{C}$ in an incubated with $5 \% \mathrm{CO}_{2}$ then changed the medium to serum free media with difference concentration of SBP extracts for 48 hours. After treatment, dscarded the medium and washed twice with cold PBS, $100 \mathrm{ul}$ of MTT solution $(5 \mathrm{mg} / \mathrm{ml})$ was added into each well, incubated at $37^{\circ} \mathrm{C}$ for 4 hours then measured by the ELISA reader at $420 \mathrm{~nm}$.

\section{Melanin content assay}

Harvested cells were lysed in cold lysis buffer (20 mM sodium phosphate $\mathrm{pH}$ 6.8, 1\% tritonX-100, $1 \mathrm{mM}$ PMSF and $1 \mathrm{mM}$ EDTA) then centrifuged 1,200 rpm at $4{ }^{\circ} \mathrm{C}$ for 10 minutes to separate supernatant out while melanin pellets were dissolved in $200 \mathrm{ul} 1 \mathrm{~N}$ $\mathrm{NaOH}$ for 60 minutes at $80{ }^{\circ} \mathrm{C}$. ELISA reader were used for measuring the absorbance at $415 \mathrm{~nm}$. and calculated compared to the protein content from bicinchoninic acid (BCA) protein content assay (Pierce Bitechnology, Packford, IL, U.S.A).

\section{Western blot analysis}

Supernatant that was separated during progression of cell lysis was be taken to fractionate on SDS-PAGE then transferred to Hybond enhanced chemiluminescence nitrocellulose membrane (mersham, Little Chalfont, UK) and probe with primary antibody against tyrosinase (Santa Cruz, Biotechnology, Santa Cruz, CA, U.S.A), MITF ( Merck Millipore Darmstadt, Germany), ERK (Merck Millipore Darnstadt, Germany), P38 ( Merck Darmstadt Millipore, Germany), and monoclonal antibodies against B-Actin (AC-15, Sigma Aldrich). From then on, incubate membrane with Horseradish peroxidaseconjugated secondary antibody complex visualization and quantified the signals by ImageJ software.

\section{Statistical analysis}

All information was be analyzed by mean \pm standard deviation (SD). The one way ANOVA test is used to measure the different between each set of information. P-value that less than 0.05 was considered as statistically significant.

\section{Results}

\section{Phenolic compound}

The objective of this experiment was to measure the efficacy of the solvents which used for extraction and also method which can extracts phenolic compound as much as possible. Most of phenolic compounds dissolve very well when extract with high polar solutions such as water, methanol $(\mathrm{MeOH})$, 
ethanol $(\mathrm{EtOH})$, acetone, ethyacetate, propanol, acetic acid or their mixture in various portions [11]. Figure 1A show that W60 can extract phenolic compound as much as $16.24 \mu \mathrm{g} / \mathrm{ml}$ followed by M60, E4 and MW24 with total phenol 13.89, 9.21 and $8.38 \mu \mathrm{g} / \mathrm{ml}$ respectively. Cytotoxicity of SBP was experimented by MTT reduction assay which measured the reduction environment from mitochondrial function of living cells by measuring the formazan formation to access cytotoxic effects. The result found that SBP at indicated concentrations (up to $500 \mu \mathrm{g} / \mathrm{ml}$ ) with 48 hours incubation. Cytotoxicity shows no significant effect after SBP treatment (Fig. 1B).

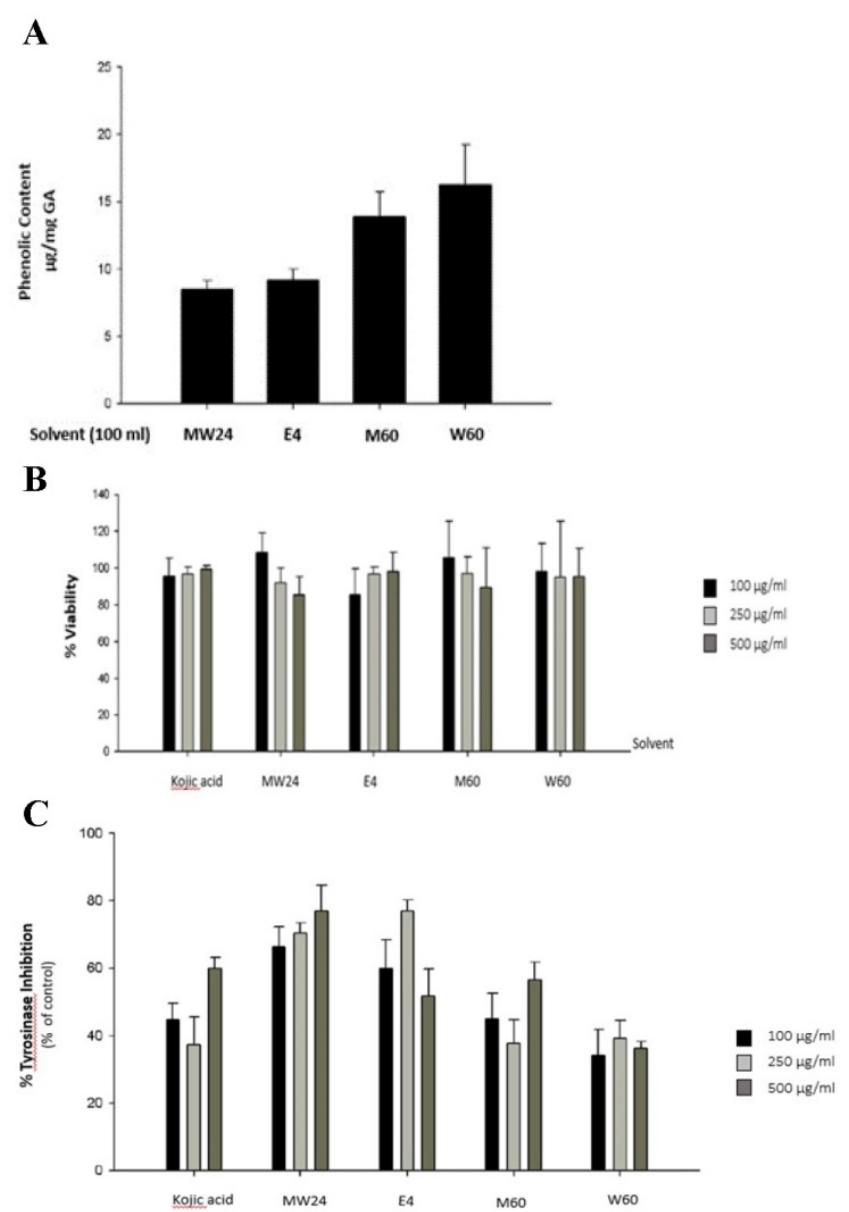

Figure 1. The effects of Sucrier banana peel (SBP) on tyrosinase activity. (A) Total phenolic compound content of Sucrier banana peel (SBP) from different extracted solvent. (B) Effects of SBP on cell viability and (C) tyrosinase inhibition percentage after treated with indicated concentrations of SBP.

\section{The effects of SBP on enzyme activity and melanin content in B16F10 cells}

To test melanogenesis suppression of SBP, first step was to test capability of mushroom tyrosinase enzyme inhibition compare to kojic acid which is commercial whitening agent. SBP (MW24) has higher efficacy than Kojic acid and other extractions at the same concentration $(100-500 \mu \mathrm{g} / \mathrm{ml})$. MW24 has highest percentage of tyrosinase inhibition (66.39$77.05 \%)$ while kojic acid only has percentage of tyrosinase inhibition at $44.68-59.93 \%$ as shown in Fig. $1 \mathrm{C}$. From then on, MW24 with highest percentage of tyrosinase inhibition was selected for antimelanogenesis measurement by melanin production from B16F10 cells. The melanin content of B16F10 cells was reduced after SBP (MW24) treatment (Fig. 2A).The amount of melanin content of B16F10 cells decrease significantly after stimulated with a-MSH and treated with SBP (MW24) for 24 hours in dose dependent manner as shown in Fig. 2B.

\section{Effects of SBP (M24) on the protein expression levels of tyrosinase and MITF}

Western blot analysis was used for tyrosinase and MITF activity by measuring protein expression levels after treated with SBP (MW24) for 24 hours. Regulation of melanogenic enzyme such as TRP-1, TRP-2 resulted from transcriptional effect of MITF and tyrosinase protein level. The results shows that SBP (MW24) was capable of dose dependent down-regulate both tyrosinase and MITF protein expression as shown in Fig 3.

\section{Effect of SBP (M24) on melanogenesis inhibition through mitogen-activated protein kinase (MAPKs) signaling pathway}

In melanogenesis, MAPKs kinase family especially ERK and p38 are related directly with this procedure [12]. The ERK signaling will down-regulate while p38 signaling will up-regulate during occurrence of melanin production. However, phosphorylation of ERK and p38 will have reverse procedure of melanin production. The results showed clearly effect after SBP (MW24) treatment with a-MSH activated by immunoblotting to MAPK signaling pathway. SBP (MW24) slightly decreased p38 protein expression and up-regulate phosphorylation of p38. This result showed the way to inhibit melanogenesis process through p38 pathway without any effect to ERK but phosphorylation of ERK protein level slightly decreased after a-MSH and SBP (MW24) treatment as showed in Fig. 4.

\section{Discussion}

Sucrier banana peels are agricultural wasted that have abundance of many active ingredient substances especially in the group of phenolic compounds and carotenoid which are the secondary metabolite that plants create for self-defense mechanism [7]. These substances can be found in leaves, barks, fruits, peels, seeds of almost all plants. Phenolic compounds and carotenoids are phytochemical substances which have 
good properties for human health. The substances comprise anti-inflammatory, anti-viral, analgesic, antioxidant, anti-carcinogen and etc [13]. In general, phenolic compounds can be well dissolved in high polar solvent. For experiment, extraction of MW24 should be able to extract phenolic compound the most because of procedure of extraction from Folin-Cicualteu assay. Low polar compounds was be extracted first then follow by high polar substances depending on final mixing ratio of solvent in the procedure. The results show that W60 can extract phenolic compounds the most, possibly due to 60 degree Celsius temperature which could be the appropriate temperature to extract phenolic compounds [11]. It can also be assumed that in SBP may contain high polar substances which dissolve well in water. When compare the amount of total phenolic compounds as shown in Fig. 1A, W60, M60, E4 and MW24 demonstrated the possibility of substance solubility, significantly.

After total amount of phenolic compound was known, we can proceed to pinpoint type and amount of flavonoid and carotenoid substances. This we found SBP (MW24) extracts contain ferullic acid as high as $906.62 \mathrm{mg} / 100 \mathrm{~g}$ and also found small amount of lutein and $\beta$-carotein which categorized in carotenoid group at 1.23 and $2.37 \mathrm{mg} / 100 \mathrm{~g}$ respectively. Through SBD (MW24) still has high percentage of tyrosinase inhibition. Therefore, SBP (MW 24) was be selected for the further studied.

From cytotoxicity testing, no significant cytotoxic was found from SBP although amount of usage concentration was up to $500 \mu \mathrm{g} / \mathrm{ml}$. possibly because of the small amount of carotenoid within extraction has the capability of protecting cells. Substances in this group have antioxidant properties which can terminate poison through Cytochrome P-450 especially $\beta$-carotein that has stimulating role of immune functions by interacting with Reaction Oxygen Species (ROS). This will support effectiveness to phenolic compound which has ROS scavenging effect property to decrease inflammatory cytokine [14]. So, SBD (MW24) not only has no toxic effect, it has the role of cell protection as well.

Our study also found that SBP (MW24) has capability of inhibiting melanogenesis of B16F10 cells. The expression of tyrosinase and MITF were reduced after SBP (MW24) treatment in both assessed with mushroom tyrosinase and Western blot analysis in a dose dependent manner. This test clearly indicated that SBP (MW24) can suppress the creation of melanin by down-regulate p38 signaling pathway and up-regulate phosphorylation of p38 which activate MITF protein degradation then effect to decrease the expression of melanogenic enzyme family such as tyrosinase. Suppression of p38 decreases to cAMP Response Binding Protein (CREB) functions which is an important transcript factor that works with PAX3 and SOX10 that attaches to M-BOX of MITF gene to activate MITF protein expression that effect to melanin production process and cells survival activity [15]. In general, activating of ERK leads to phosphorylation of MITF at serine 73 which causes ubiquitination and eventually degradation. Most of natural compounds can inhibit melanogenesis through the stimulation process of ERK signaling pathway, but SBP (MW24) was not significantly influence ERK signaling pathway [16].

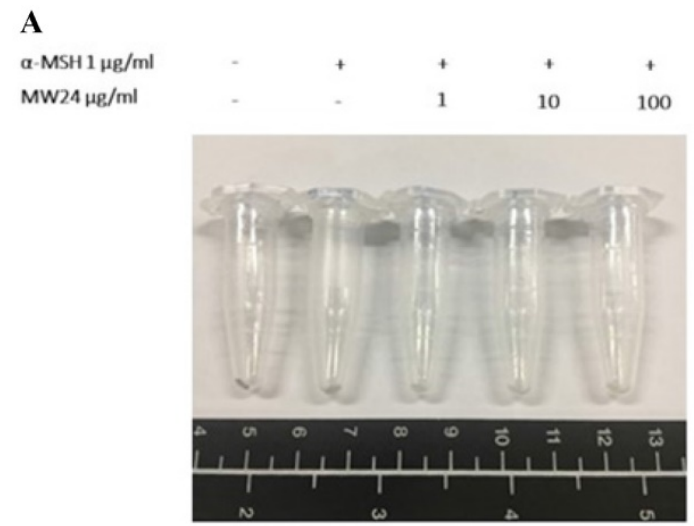

B

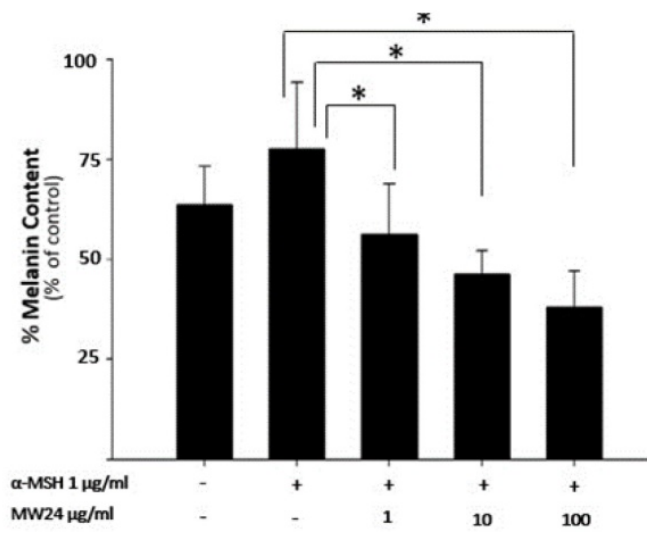

Figure 2. Effects of SBP on melanin production in Bl6F10 cell. (A) Photograph of precipitated B16F10 cells after treatment of SBP $(1-100 \mu \mathrm{g} / \mathrm{ml})$ with $\alpha-\mathrm{MSH}$ for 24 hours. (B) Melanin production in B16F10 cells. (mean \pm SD, n=7) $* P<$ 0.01 .

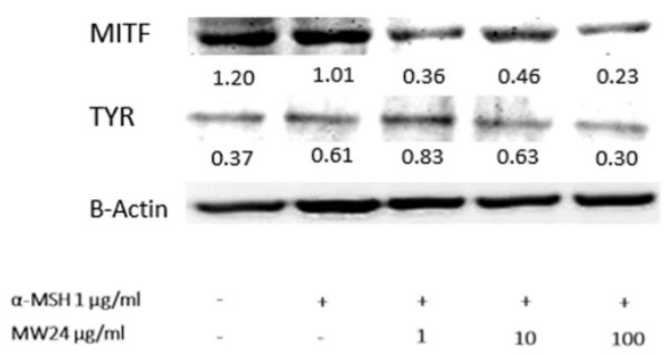

Figure 3. Tyrosinase and MITF expression levels of after SBP treatment. Cells were treated with SBP at the concentration of 1,10 or $100 \mu \mathrm{g} / \mathrm{ml}$ for $24 \mathrm{~h}$. Western blot analysis was used for protein expression determination and $\beta$-actin was used for comparison of protein expression by Image] software. 


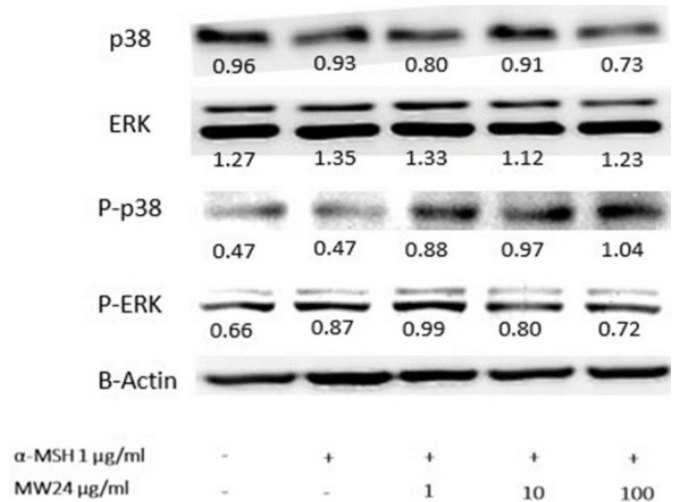

Figure 4. SBP (MW24) inhibited melanin production through ERK pathway. Primary B16F10 cells were treated with SBP (MW24) at the concentration of $100 \mu \mathrm{g} / \mathrm{ml}$ for 24 hours $\beta$-actin the quantitative control was used for relative proteins expression comparison as shown by inserted number.

Many research found that phenolic compounds and carotenoid can inhibit the production of melanin pigments because of beta-carotene has the capability of absorbing UVB photon while also has the property of the lipid soluble antioxidant and phenolic compound can activate Nrf2, block ROS receptor, decrease inflammatory cytokine production and induce y-GSC expression which support to our study but in the difference way of melanogenesis inhibition [17]. Other than that ferulic acid found in SBP (MW24) was the main substance within group of phenolic compounds confirmed by several studies that it has the property of anti-melanogenesis because it can modulate vascular endothethelial growth factor (VEGF) expression, induces nitric oxide synthase, act as tumor suppressor gene and also relate to the procedure of melanin pigments production. In SBP (MW24) solution, ferulic acid can increase effectiveness when mix with carotenoid or other phospholipids because the water solubility, lipid solubility and bioavailability will be increased resulted to improve the melanogenesis inhibition of B16F10 cells [18]. The implication of all the results concludes that SBP (MW24) could reduce melanogenesis effectively.

\section{Abbreviations}

MITF: microphthalmia associated transcription factor; a-MSH: a-melanocytes stimulating hormone; MC1R: melanocortin-1 receptor; TRP1: tyrosinaserelated protein 1; TRP2: tyrosinase-related protein 2; MAPK: mitogen-activated protein kinase.

\section{Acknowledgements}

This work was supported by grants from the Royal Golden Jubilee Ph.D.: RGJPHD. Thailand. (PHD/0017/2558).

\section{Author Contributions}

R.H., K.T., K.S. and U.P. conceived and designed the experiments; R.H. C.H.L. and M.C. performed the experiments; R.H., and M.C. and C.H.L analyzed the data; R.H. contributed reagents/materials/analysis tools; R.H. and C. H. L.wrote the paper.

\section{Competing Interests}

The authors have declared that no competing interest exists.

\section{References}

1. Regnault MR, Gadaud N, Boulinguez S, Tournier E, Lamant L, Gladieff L, et al. Chemotherapy-related reticulate hyperpigmentation: a case series and review of the literature. Dermatology 2015; 231:312-8.

2. Ali A. Dermatology A Pictorial Review. Mc Graw Hill Education 2010. ISBN978-0-07-179323-0.

3. Garraway LA, Widlund R, Rubin MA, Getz G, Berger AJ, Ramaswamy S, Beroukhim $\mathrm{R}$, et al. Integrative genomic analyses identify MITF as a lineage survival oncogene amplified in malignant melanoma. Nature 2005; 436: 117-22.

4. Busca R, Ballotti R. Cyclic AMP a key messenger in the regulation of skin pigmentation. Pigment cell and Malanoma Research 2000; 13: 60-9.

5. Zhou J, Ren T, Li Y, Cheng A, Xie W, Xu L, et al. Oleoylethanolamide inhibits a-melanocyte stimulating hormone stimulated melanogenesis via ERK, Akt and CREB signaling pathways in B16 melanoma cells. Oncotarget 2017; 8: 56868-79.

6. Onkoksoong $\mathrm{T}$, Jeayeng $\mathrm{S}$, Poungvarin $\mathrm{N}$, Limsaengurai $\mathrm{S}$, Thamsermsang $\mathrm{O}$, Tripatara $\mathrm{P}$, Akarasereenont $\mathrm{P}$, Panich U. Thai herbal antipyretic 22 formula (APF22) inhibits UVA-mediated melanogenesis through activation of Nrf2-regulated antioxidant defense. Phytotherapy Research 2018; 32: 1546-54.

7. Singh B, Singh JP, Kaur A, Singh N. Bioactive compounds in banana and their associated health benefits - A review. Food Chemistry 2015; 1: 1-11.

8. Leerach N, Yakaew S, Phimnuan P, Soimee W, Nakyai W, Luangbudnark W, Viyoch J. Effect of Thai banana (Musa AA group) in reducing accumulation of oxidation end products in UVB-irradiated mouse skin. Journal of Photochemistry \& Photobiology, B: Biology 2017; 168: 50-58.

9. Hung BY, Kim SY, Jung JM, Won CH, Choi JH, Lee MW. The antimycotic agent clotrimazole inhibit melanogenesis by accerlerating ERK and PI3K-/Akt-mediated tyrosinase degradation. Experimental Dermatology 2015; 24: 381-400.

10. Shen T, Heo SI, Wang MH. Involement of the p38 MAPK and ERK signaling pathway in the anti-melanogenic effect of methyl 3,5- dicaffeoyl quinqte in B16F10 mouse melanoma cell. Chemico-Biological Interaction 2012; 199: 106-11.

11. Ruesgas-Ramón M, Figueroa-Espinoza MC, Durand E. Application of Deep Eutectic Solvents (DES) for Phenolic Compounds Extraction: Overview, Challenges, and Opportunities. Journal of Agricultural and Food Chemistry 2017; 10: 3591-3601.

12. Tsao YT, Kuo CY, Kuan YD, Lin HC, Wu LH, Lee CH. The Extracts of Astragalus membranaceus Inhibit Melanogenesis through the ERK Signaling Pathway. International Journal of Medical Sciences 2017; 3: 1049-53.

13. Chang TS. An updated review of tyrosinase inhibitors. International Journal of Molecular Sciences 2009; 26: 2440-75.

14. Juturu V, Bowman JP, Deshpande J. Overall skin tone and skin-lightening-improving effects with oral supplementation of lutein and zeaxanthin isomers: a double-blind, placebo-controlled clinical trial. Clinical, Cosmetic and Investigational Dermatology 2016; 7: 325-332.

15. Bell RE, Levy C. The three m's: melanoma, micro-phthalmia-associated transcription factor and microRNA. Pigment Cell Melanoma Research 2011; 24: 1088-106.

16. Zhou J, Ren T, Li Y, Cheng A, Xie W, Xu L, Peng L. Oleoylethanolamide inhibits a-melanocyte stimulating hormone-stimulated melanogenesis via ERK, Akt and CREB signaling pathways in B16 melanoma cells. Oncotarget 2017; 23: 56868-79.

17. Toews DPL, Hofmeister NR, Taylor SA. The Evolution and Genetics of Carotenoid Processing in Animals. Trends in Genetic 2017; 33: 171-182.

18. Li L, Liu Y, Xue Y, Zhu J, Wang X, Dong Y. Preparation of a ferulic acid-phospholipid complex to improve solubility, dissolution, and B16F10 cellular melanogenesis inhibition activity. Chemistry Central Journal 2017; 22: $11-26$. 